\title{
Investigation and Research on the Inheritance Status of Original Ecological Yao Folk Songs in Ruyuan Yao Autonomous County, Guangdong Province
}

\author{
Qunying Wang ${ }^{1, *}$ Yongwei Liu $^{1}$ \\ ${ }^{1}$ College of Music and Dance, Shaoguan University, Shaoguan, Guangdong 512005, China \\ *Corresponding author. 2568264195@qq.com
}

\begin{abstract}
Yao folk songs, with their long and fine sound, reflect the historical accumulation and spiritual culture of the Yao people in Ruyuan, and are of great value in humanistic research. However, the inheritors of Yao folk songs of Ruyuan are getting older and the way of inheritance is relatively simple. This paper will investigate the inheritance status of Yao folk songs in Ruyuan Yao Autonomous County, Guangdong Province. This will include the status quo of the inheritor, the way of inheritance, the task of inheritance, the repertoire of inheritance and the problems in the process of inheritance, etc., in order to have a more comprehensive understanding of the inheritance status of Ruyuan Yao folk songs.
\end{abstract}

Keywords: Yao folk songs in Ruyuan, inheritance status, inheritance and development

\section{INTRODUCTION}

Ruyuan Yao Autonomous County is located in the adret of the Nanling Mountains. Everyone of Yao nationality enjoys singing folk songs and is good at it. In daily life, Yao people sing songs all the time. Whenever they are working in the fields, meeting each other daily, going hunting up the mountain and attending weddings, etc., they talk with each other through songs. And on New Year's Day or other festivals, Yao people hold a series of activities where they sing and dance to express the festive and good wishes through songs. The folk songs of Ruyuan Yao nationality reflect the historical precipitation and spiritual culture of the people of Ruyuan Yao nationality and are thus of high research value. However, the inheritance and development of Ruyuan Yao folk songs is facing a crisis, which deserves attention and research.

\section{THE STATUS QUO OF THE INHERITORS OF}

\section{RUYUAN FOLK SONGS}

\section{A. Investigation of the inheritors}

Inheritors are the soul in the process of immaterial cultural heritage inheritance, because it is their

*Fund: Phased achievement of the innovation project for college students in 2019 - "Ecological Inheritance of Ruyuan Yao Folk Songs from National Intangible Cultural Heritage" (Project No.: 201910576017); phased achievement of the teaching reform project of higher education in Guangdong province in 2018 — "Construction of Innovative System of Practice Teaching of Music Collecting Style in Local Colleges and Universities". existence that makes the culture go ahead. Here is an overview of information about the inheritors of Ruyuan Yao folk songs ("Table I"). 
TABLE I. OVERVIEW OF INFORMATION ABOUT THE INHERITORS OF RUYUAN YAO FOLK SONGS

\begin{tabular}{|c|l|l|}
\hline Name & Level & \multicolumn{1}{c|}{ Status quo and related information } \\
\hline $\begin{array}{c}\text { Zhao } \\
\text { Xinrong }\end{array}$ & $\begin{array}{l}\text { National } \\
\text { level }\end{array}$ & $\begin{array}{l}\text { Female, 72 years old. With a love for them since little, at the age of 6, she began learning to sing the } \\
\text { songs from her grandfather who was a private adviser" then, and could sing a lot of them at 10. She is } \\
\text { now active in variou art occasions and often teaches in the Cultural Center of Ruyuan Yao Autonomous } \\
\text { County. }\end{array}$ \\
\hline $\begin{array}{c}\text { Zhao } \\
\text { Caifu }\end{array}$ & $\begin{array}{l}\text { Provinci } \\
\text { al level }\end{array}$ & $\begin{array}{l}\text { Male, 54 years old He is now active in various art occasions and often teaches in the Cultural Center of } \\
\text { Ruyuan Yao Autonomous County. }\end{array}$ \\
\hline $\begin{array}{c}\text { Deng } \\
\text { iangyin }\end{array}$ & $\begin{array}{l}\text { County } \\
\text { level }\end{array}$ & $\begin{array}{l}\text { Female, 56 years old She used to listen to elders singing when little but have never learned to sing the } \\
\text { songs in any formal way due to her job. She began to learn from the national-level inheritor of Yao Folk } \\
\text { songs after retirement. She is now active in various art occasions and often learns and teaches in the } \\
\text { Cultural Center of Ruyuan Yao Autonomous County. }\end{array}$ \\
\hline $\begin{array}{c}\text { Zhao } \\
\text { Liangman }\end{array}$ & $\begin{array}{l}\text { County } \\
\text { level }\end{array}$ & $\begin{array}{l}\text { Male, 56 years old He is now active in various art occasions and often teaches in the Cultural Center of } \\
\text { Ruyuan Yao Autonomous County. }\end{array}$ \\
\hline
\end{tabular}

Currently the inheritors of Ruyuan Yao Folk Songs are "national- level Zhao Xinrong, provincial-level Zhao Caifu, county-level Deng Xiangyin and countylevel Zhao Liangman", all of which are advanced in years above 50. With the change of time, Yao folk songs are on the verge of disappearing. Inheritors of intangible cultural heritage play a critical role in the process of inheriting. Therefore, it has become an important measure to pay attention to the dynamics of the inheritors, which is worth people's concern.

\section{B. Goals and tasks of the inheritance}

In many modernized countries, "inheritors of intangible culture" are called "national treasure in life". With the development of China, the intangible cultural inheritors have been paid more and more attention by the country and the people. The first issue to consider is to establish the goals and tasks of the inheritance. The national-level inheritor of Ruyuan Yao folk songs Zhao Xinrong said, With "inheritance and development" as the primary goal and task, in cooperation with the work arrangement, teaching and performance of the Cultural Center, it is necessary to inherit Yao folk songs through the form of teacher-student impartation, so that more people can learn Yao folk songs and sing them. Meanwhile, coupled with related measures launched by the local cultural center, it is also important to collect and sort out Yao songs in order to make innovations and endow them with the meaning of the new era. And creating Yao songs of the new era is an important direction of inheritance goal.

"Remaking the Yao folk songs" is an important measure to realize this idea. The content of Yao folk songs is not rich enough, mostly about trifles in life and eulogizing mythological figures, in elusive language, which causes the failure of Ruyuan Yao folk songs to be extensively spread. "Remaking the Yao folk songs" not only retains the characteristics and essence of Yao folk songs but also combines the contents of the new era. By the translation of Yao language, the language barrier is removes, the content is enriched and they are transmitted more widely, representing the features and charm of Yao folk songs in the new era".

\section{The supportive policies of the government}

In the inheritance and development of intangible cultural heritage, the relevant support policies of the government and its economic support for the inheritors play a very important role. According to Zhao Xinrong, since the confirmation of my identity as an inheritor, related government departments have paid great attention to me. They dole out a certain amount of money every year and encourage me to teach and participate in performances in the cultural center, schools and other occasions. The ways and channels of inheritance are becoming more and in a wider range. I feel the attention of the government and its effort of support that has protected the subject of the inheritance, which is a significant factor in the inheritance and development of Ruyuan Yao folk songs.

\section{THE INHERITANCE STATUS OF RUYUAN FOLK SONGS}

\section{A. Ways of inheritance}

1) Inheritance through traditional festivals such as the "Shiyue Zhao" Festival" (the first day of the tenth solar month) and "the Panwang Festival", etc.: The "Shiyue Zhao" Festival" is a traditional Yao festival (in the custom of Yao nationality, the first day of a month is called "zhao"), symbolizing a year of grain harvest and the arrival of a bumper harvest. Zhao Xinrong said, " The 'Shiyue Zhao Festival' each year is a grand day for the Yao people. To celebrate it, they hold activities for three days and three nights. And as a Yao song singer, I also have to sing for three days and three nights. The diverse activities on the scene also attract tourists and scholars from around the country, and those love Yao songs among them will come and learn a bit from me." And the "Panwang Festival" is another grand festival to worship Panwang, a ceremony of special 
significance for Yao people. To celebrate it, the "master" holds the activity of paying tribute to Panwang, where the Yao people sing "Panwang Song" and dance "Panwang Dance" to commemorate Panwang. And at these festivals, people will put on Yao clothing, sing Yao songs and dance Yao dances. Therefore, the grand festivals held each year is an important channel of Yao song inheritance.

2) Inheritance through written music scores: In order to make Ruyuan Yao songs be inherited lively, and intensify the efforts of inheriting Yao culture, the local government and cultural center carried out a series of activities such as "Bringing Yao song and dance into the campus" in combination with the situation of the school. The "Yao song and dance" was also included in the curriculum of the school. Combined with the age of students and the difficulty of the Yao language, teaching plans and school-based teaching textbooks have been compiled, and inheritors are arranged to give lectures themself. In this way, the folk treasure can be inherited by elementary school students, so that "the song and dance of the Yao nationality" can truly enter the campus, contributing to the full preparations for training a new generation and more inheritors. The local bureau of publication of cultural radio, film and television news of Ruyuan Yao autonomous county also published the "Selection of Folk Songs of Ruyuan Yao Nationality", which collected and sorted out a large number of local folk songs. They are of many categories: the type of "myth, legend, migration"; the type of "love, marriage, family; the type of "production, life, knowledge". The writing of music and books enables people to have a deeper and clearer understanding of the folk songs of the Ruyuan Yao nationality, which is an important medium for the inheritance of the folk songs of the Ruyuan Yao nationality.

3) Inheritance through image data: With the departures of inheritors, the rich cultural heritage of Yao folk songs will also fade. In order to better realize the protection and inheritance of Ruyuan Yao folk songs, relevant experts and scholars as well as local cultural departments have made a lot of preparations. They have recorded the folk songs of Ruyuan Yao nationality, and recorded them on CD. Many CDS have come to the hands of the inheritors, and there are also recordings of Yao folk songs available for appreciation in the local World Guoshan Yao Museum. In addition, many books on Yao folk songs have been published and some are being written. In particular, the curator of the local cultural center, Pan Guiqing, adapted the original Yao song with the elements of the new era, forming a new form of "remaking the Yao folk songs" and relevant audio has been preserved. And there are also many pieces of audio and videos of Yao folk songs online, which enable more people to understand Yao folk songs, and also let more young people pay more attention to them.

4) Inheritance through media: Newspapers, magazines, radio, television, the Internet, WeChat and other mass media have quietly integrated into every aspect of people's lives. TV, Internet and other media truly show the majority of viewers and Internet users the original Ruyuan Yao folk culture, which improves the efficiency of the spread Ruyuan Yao nationality folk songs and allows more people to get to know and then understand Ruyuan Yao nationality folk songs, folk songs. A large number of people are attracted by these media to come to Ruyuan to experience the charm of Yao folk songs. In recent years, the folk songs of the Yao nationality have aroused the attention of many people, and there have been a number of media going deep into Ruyuan to make interviews. Under the media reports, Ruyuan Yao folk songs have been known by more and more people, and attracted many experts, scholars, students and Yao song lovers to come to Ruyuan to investigate and study Yao folk songs, which promoted the inheritance and development of them. Therefore, media inheritance has also become a bridge facilitating the folk songs of the Ruyuan Yao nationality to go out and allowing the outside world to know and understand the folk songs of the Ruyuan Yao nationality.

\section{B. Repertoire of inheritance}

There are not many songs with scores among the inherited ones and they are transmitted orally mainly as most of the songs have only lyrics, with no particular personal writing them down in the form of scores. Later, Pan Guiqing, curator of Ruyuan cultural center, arranged the original ecological Yao folk songs. And the "Selection of Folk Songs of Ruyuan Yao Nationality" compiled by the bureau of culture, radio, television, press and publication of Ruyuan Yao Autonomous County collects the folk songs sung for words in occasions of toiling and hunting, festival activities, weddings, friends and family gatherings, dinner parties and holding religious ceremonies etc. by Ruyuan Yao people through the ages. Among the inherited songs, the "Song of Panwang of Yao Nationality" and "Great Song of Panwang" are the worship of the Yao ancestor, Panwang, which praise the story of the national savior "Pan Hu Legend" and are widely sung folk songs. The "Flowers in the Twelve Months" is a song made through praising the beauty of flowers across the twelve months and referring to them in the time sequence of their blossom to express people's joy of harvest. "Old Folk Song of Yao 
Nationality" and "Song of Migration", etc. are songs about migration.

In addition to the above much sung songs, there are many on other themes, such as the type of "myth, legend, migration" represented by "The Birth of Panwang", "Song of Migration" and "The Great Drought", etc., the type of "love, marriage, family", represented by "Nightfall", "Teaching the Bride and Bridegroom to Sing" and "Toasting Song for Wedding", etc., as well as the type of "production, life, knowledge" represented by "Spring Ploughing", "Children of Yao passing on the Songs" and "Song for the Twelve Chinese Zodiac Signs", etc.

Zhao Xinrong not only knows very well how to pass the songs down, being able to sing some of the songs passed down from generation to generation, but also make impromptu performance upon what she sees and thinks in life. From the folk songs, one can understand the lives of the Yao people and their history and culture.

\section{Problems in the inheritance of Yao folk songs}

1) Young people only have average interest in them: Among the young people, the degree of love for Yao folk songs is average. As the times advancing, with the impact of popular music, although Yao songs are close to life and have their own artistic characteristics and have gained a certain influence in the broad masses, from the overall point of view, young people only have average interest in them. In addition, quite a few young people think that Yao song is too vulgar and backward.

2) The media publicity is weak and the role of school inheritance should be strengthened: In an age when information travels so fast, the voice of the media is everywhere. As the carrier of information transmission, the media plays an important role in promoting and inheriting culture, especially in the propaganda of books, magazines, radio, newspapers, TV and Internet. , Without enough publicity of the media, the Ruyuan Yao folk songs needs further strengthened propaganda work. School inheritance plays an important role in the inheritance of Yao folk songs. In the investigation, it is learned that although there are school-based courses under "bringing Yao songs schools" school-based curriculum in primary and secondary schools, they were suspend after some time due to low attention paid to them. Therefore, the inheritance role of schools needs to be strengthened.

3) The age of the audience is high and the range of target audience needs to be broadened: Yao folk songs have a long history. In the investigation and interview, it is found that among the audiences of the Ruyuan Yao folk songs, the elderly are the main body, and only a small number of them are young people. As the cultural essence of the Ruyuan Yao nationality, the folk songs of the Ruyuan Yao nationality should be extended to the broad masses and get a broader range of target audience.

4) The content is not rich enough, with insufficient creativity: According to the inheritor and curator of the heritage and cultural center, based on conversations in daily life, with mainly impromptu lyrics, most Yao folk songs in Ruyuan have scant contents, and most of the performance are in the form of solo singing and duet singing, which is too simple; in addition, since most of them are sung in original Yao dialect, the audience inevitably think that the Ruyuan Yao song is low and unrefined. Compared with the Yao folk songs in Guangxi and other places, the situation of the Yao folk songs in Ruyuan is not optimistic. The contents of Yao folk songs in Guangxi and other places are rich, not limited to the art itself. Through artistic procession, efforts are made to make innovation in performance forms. And it is the goal of the future efforts of Ruyuan Yao folk songs to improve the innovation ability, create more diversified contents and enhance the artistic taste.

5) Activities fall to formality and systematic implementation to be strengthened: The implementation of the activities is of far-reaching significance to the development of Ruyuan Yao folk songs. According to the interview, the activities related to the folk songs of the Ruyuan Yao nationality fall to formality and are just slipped by; relevant activities are carried out without combining the features of the new era to enrich the contents and change the ways of activities. Therefore, the systematic implementation needs to be strengthened.

6) The language barrier of the folk songs: Language is a major obstacle for inheriting and innovating the folk songs of the Ruyuan Yao nationality. As a very unique language, the Yao dialect has a very wide range of pronunciations. Yet since it has no characters, there is no related written record. There are more than 30 initials and more than 100 finals in Yao language, and the 8 tones, 4 more than the Chinese language. Therefore, the Yao dialect is rather difficult to learn. The complicated pronunciation of Yao language greatly limited the learning of the Yao dialect in Ruyuan. And when interviewed by the author, Deng Xiangyin, the provincial-level inheritor said, the main factor barring Yao folk songs from being transmitted widely is the language in which they are sung is different with what are used normally, leading to the incomprehension of the lyrics. As a result, people are not able to write lyrics unless they master the language as the lyrics of Yao folk songs are improvised orally. 
Therefore, the learning of Yao language is an important factor in the development of Yao folk songs.

\section{CONCLUSION}

The Yao folk songs is the reproduction of the life of Yao nationality, which contains the memory of the life of Yao nationality for generations and the spirit of Yao nationality, and it shows the temperament of this nationality to the world. This kind of culture and art which serves as a tie should arouse people's attention. In view of the current situation, efforts should be made to improve young people's cultural identity of Yao folk songs, enhance their awareness of protection, and take effective measures to diversify the ways of inheritance. At the same time, it is necessary to give full play to the advantages of school inheritance and pay attention to the talent cultivation of Yao folk song inheritance. Besides, the inheritance and development of Yao folk songs is inseparable from its own inheritance and innovation. So it is necessary not only to innovate in content and form of expression, but also to make the melody of Yao folk songs more in line with the public's aesthetic taste, keep pace with the times in content, and show the life outlook and cultural spirit of the Yao nationality in Ruyuan in the new era.

\section{References}

[1] Wang Man, Wang Qian. Study on the Status Quo, Inheritance and Protection of Jianghua Yao Folk Songs [J]. National Folk Music. 2015 (03). (in Chinese)

[2] Pan Guiqing. Thoughts on the Inheritance and Development of Ruyuan Intangible Cultural Heritage [J]. Folk Customs in China (Academic Edition), 2011 (04). (in Chinese)

[3] Li Jinyun. Stickment and Adjustment: Research on the Traditional Cultural Inheritance of Yuyuan over Mountain Yao [D]. South-Central Minzu University, 2018. (in Chinese)

[4] Zhao Xiufen. Discussion on the Inheritance and Protection of Yao Folk Songs [J]. Song of Yellow River, 2019 (18): 57-58. (in Chinese)

[5] Zhang Hongshun. Interpretation of the Musical Features of Yao Minority's Folksongs in Ruyuan, Guangdong [J]. Journal of Xinghai Conservatory of Music, 2019 (03): 105-115. (in Chinese)

[6] Zhao Xuchao. A Study on the Spatial Distribution of Yao Folk Song Singing and Migration in the Northern Part of Guangdong Province [J]. Journal of Guangxi University For Nationalities (Philosophy and Social Science Edition), 209, 41 (05): 119-125. (in Chinese)

[7] Qiu Jingya. Research on the Musical Style and Singing Art of Jianghua Yao Folk Songs [D]. Hunan Normal University, 2010. (in Chinese)

[8] Zhao Xiufen. Discussion on the Inheritance and Protection of Yao Folk Songs [J]. Sound of Yellow River, 2019 (18): 57-58. (in Chinese)

[9] Hao Qinyu. Research on the Status Quo and Problems of Representative Inheritors of Beijing Municipal Intangible Cultural Heritage Projects [D]. Chinese National Academy of Arts, 2014. (in Chinese) 